$\mathrm{Ca}^{2+}$ and are usually found in plasma membranes. Both adrenergic and cholinergic stimuli cause breakdown of these lipids ${ }^{10,11}$ which would be followed by a loss of PI for their resynthesis. Whether this could explain the PI response generally remains to be seen, since the polyphosphoinositides occur in such low concentrations in most tissues. Polyphosphoinositides are unlikely to replace PI in a calcium gating theory, because their hydrolysis seems to require calcium ${ }^{11,12}$. Nevertheless, on the basis that there is a less obvious calcium requirement for the vasopressin-induced breakdown of triphosphoinositide in hepatocytes ${ }^{13}$, Michell now seems prepared to shift his ground from PI to this lipid.

What are PI and polyphosphoinositides doing if not $\mathrm{Ca}^{2+}$ gating? There is no shortage of suggestions but none provides a convincing general explanation. Conversion of PI to diacylglycerol, for example, could produce a localized increase in membrane fluidity. This in turn could facilitate exocytosis or protein-protein interactions in a membrane. Recent work suggests that polyphosphoinositides and PI may control protein kinases ${ }^{14,15}$. Activation of some receptors leads to protein phosphorylation, so the possible involvement of inositol lipids deserves further study.

The inositol lipids are rich in arachidonic acid and another theory of the PI response is that receptor-linked hydrolysis supplies this fatty acid for prostaglandin synthesis ${ }^{16}$. Since many systems showing the PI effect do not require prostaglandins for the physiological response, this theory cannot apply generally.

It would be tedious to continue the list of inadequate answers to the puzzle of PI. If this is goodbye to the calcium gating theory, we shall miss it, at least until we have a better explanation.

1. Michell, R.H. Biochim. biophys. Acta 415, 81 (1975)

2. Hokin, M.R. \& Hokin, L.E. J. biol. Chem. 203, 967 (1953).

3. Michell, R.H. \& Kirk, C.J. Trends pharmac. Sci. 2 , 86 (1981).

4. Cockroft, S. Trends pharmac. Sci. 2, 340 (1981).

5. Farese, R.V., Larson, R.E. \& Sabir, M.A. Biochim biophys. Acta 633, 479 (1980)

6. Cockroft, S., Bennett, J.P.\& Gomperts, B.D. Nature 288 275 (1980).

7. Bell, R.J.\& Majerus, P.W. J. biol. Chem. 255, 1790 (1980)

. Fisher, S.K., Holz, R.W. \& Agranoff, B.W.J. Neurochem. 37, 491 (1981).

9. Berridge, M.W. \& Fain, J.N. Biochem. J. 178, 59 (1979).

10. Abdel-Latif, A.A., Akhtar, R.A. \& Hawthorne, J.N. Biochem, 162, 61 (1977).

11. Fisher, S.K. \& Agranoff, B.W. J. Neurochem. 37, 968 (1981)

12. Akhtar, R.A. \& Abdel-Latif, A.A. Biochem. J. 192, 783 (1980)

13. Kirk, C.J. Creba, J.A., Downes, C.P \& Michell, R.H Biochem. Soc. Trans. 9, 377 (1981)

14. Kishimoto, A., Takai, Y., Mori, T., Kikkawa, U. \& Nishizuka, Y. J. biol. Chem. 255, 2273 (1980)

15. Jolles, J., Zwiers, H., Dekker, A., Wirtz, K.W.A. \& Gispen, W.H. Biochem. J. 194, 283 (1981).

16. Marshall, P.J., Dixon, J.F. \& Hokin, L.E. Proc. natn. Acad. Sci. U.S.A. 77, 3292 (1980)

\title{
A new model for giant $H$ II shells
}

\section{from J.C. Raymond}

SEVERAL years ago it was realized that the energy injected by supernovae can determine the overall structure of the interstellar medium ${ }^{1,2}$. When it is considered that the energy flux of the wind of an early-type star is roughly one per cent of the stellar luminosity it is also apparent that over its short lifetime of a few million years, an $\mathrm{O}$ star transfers more energy to the interstellar gas through its powerful wind than it does in its supernova demise. (O stars are massive and very hot blue objects often associated with dust clouds.) Theoretical models of the bubble created by such a wind in the surrounding $\mathrm{H}$ II region have been available for more than a decade ${ }^{3,4}$ and nebulae having very low densities near the centre, such as the Rosette Nebula, have been considered examples of the phenomenon. Since $O$ stars are generally formed in groups, it is expected that the winds of the stars in a group, together with any supernovae resulting from the rapid evolution of these massive stars, will act together to produce a

J.C. Raymond is at the Harvard-Smithsonian Center for Astrophysics, 60 Garden Street, Cambridge, Massachusetts 02138. larger bubble ${ }^{5}$. It is only quite recently that observations of young star clusters and the associated shells have become sufficiently precise for a detailed comparison with the theories.

One such investigation has found excellent agreement between observations of the Cygnus Superbubble and the expanding bubble model ${ }^{6}$, but a second, by M. Dopita and colleagues, argues against the straightforward expansion model for an object in the Large Magellanic Cloud? The latter group favours a model in which the stellar winds balance the collapse of the neutral hydrogen cloud from which the stars were formed ${ }^{8}$. In this model, the star cluster is offset from the centre of the collapsing cloud with the result that the standing shock wave created in the stellar wind is oblique. The normal component of the wind velocity is thermalized, providing the pressure to balance the collapsing cloud, while the tangential component becomes a flow along the inner surface of the shell. This flow produces velocity shifts which are largest close to two opposite sides of the bubble, with a band of nearly zero doppler shift (purely tangential velocity) across the middle. The model is similar to one recently proposed for the origin of Herbig-Haro objects from the winds of $T$ Tauri stars9.

Dopita et al. have made a wide variety of observations of N70, a giant filamentary shell in the Large Magellanic Cloud. It is a hollow shell $110 \mathrm{pc}$ in diameter containing the $\mathrm{O}$ association Lucke 114. Spectra of nine stars in the cluster and spectra and surface photometry of the shell itself, along with radio observations, demonstrate that photoionization by the stars accounts for the observed emission in the optical lines. Neither radio nor optical evidence supports the earlier suggestions that the shell is an old supernova remnant.

There are two arguments against the model of the standard expanding bubble created by the stellar winds - the age of the cluster, about $6 \times 10^{6}$ years based on the stellar spectra, and the pattern of velocities in the shell, with doppler splittings up to $90 \mathrm{~km} \mathrm{~s}^{-1}$. While the inferred mass loss rates of the cluster stars are adequate to produce a bubble of the observed size with an expansion velocity of $40-50 \mathrm{~km} \mathrm{~s}^{-1}$, its age would be less than one million years. Unless something like the effect of mass loss on the evolution of the cluster stars caused an order of magnitude overestimate of the age of the cluster, the observed doppler velocities of the shell are not due to overall expansion.

The second argument against the expansion picture is the pattern of velocity splittings. A nice correlation is found between the observed velocity splittings and those predicted by a model which fits the slightly non-circular shape of the nebula. The primary evidence against expansion comes from several points close to the centre of the nebula which show very little velocity splitting, though the splitting should be largest near the nebula centre if the shell is expanding. In the model of flow along the bubble surface, this is the region where the flow is almost entirely transverse to our line of sight. To rescue the expansion hypothesis it is necessary to place a high density cloud in this section of the shell to inhibit the acceleration. Such a cloud might be consistent with the high surface brightness observed in that region.

If the new model of flow along the inner boundary of the shell is correct, a reinterpretation of observations of many giant and supergiant shells in the Large Magellanic Cloud and other galaxies will be required.

1. Cox, D.P. \& Smith, B.W Astrophys, J. Lett. 189, L105 (1974)

2. McKee, C.F \& Ostriker, J.P. Astrophys, J 218, 148 (1977)

3. Pikelner, S.B. \& Shcheglov, P.V. Sov. Astr. 12, 757 (1969).

4. Castor, J., McCray, R. \& Weaver, R. Astrophys. J. Lett. 200, L107 (1975).

. Bruhweiler, F.C., Gull, T.R., Kafatos, M. \& Sofia, S. Astrophys. J. Lett. 238, L27 (1980)

6. Abbott, D.C., Beiging, J.H. \& Chruchwell, E. Astrophys. J. 250, 645 (1981)

7. Dopita, M.A., Ford, V.L., MacGregor, P.J., Mathewson, D.S. \& Wilson, I.R. Astrophys. J. 250, 103 (1981).

8. Dopita, M.A. Astrophys. J. 246, 65 (1981).

9. Canto, J. \& Rodriguez, L.F. Astrophys. J. 239, 982 